\title{
Neurodegeneration in SCA14 is associated with increased PKC $\gamma$ kinase activity, mislocalization and aggregation
}

\author{
Maggie M. K. Wong ${ }^{1}$, Stephanie D. Hoekstra', Jane Vowles², Lauren M. Watson', Geraint Fuller ${ }^{3}$, \\ Andrea H. Németh ${ }^{4,5}$, Sally A. Cowley ${ }^{2}$, Olaf Ansorge ${ }^{4}$, Kevin Talbot ${ }^{4}$ and Esther B. E. Becker ${ }^{1 *}$ (D)
}

\begin{abstract}
Spinocerebellar ataxia type 14 (SCA14) is a subtype of the autosomal dominant cerebellar ataxias that is characterized by slowly progressive cerebellar dysfunction and neurodegeneration. SCA14 is caused by mutations in the PRKCG gene, encoding protein kinase C gamma (PKC $)$ ). Despite the identification of 40 distinct disease-causing mutations in PRKCG, the pathological mechanisms underlying SCA14 remain poorly understood. Here we report the molecular neuropathology of SCA14 in post-mortem cerebellum and in human patient-derived induced pluripotent stem cells (iPSCs) carrying two distinct SCA14 mutations in the C1 domain of PKC $\gamma$, H36R and H101Q. We show that endogenous expression of these mutations results in the cytoplasmic mislocalization and aggregation of PKC $Y$ in both patient iPSCs and cerebellum. PKCY aggregates were not efficiently targeted for degradation. Moreover, mutant PKC $\gamma$ was found to be hyper-activated, resulting in increased substrate phosphorylation. Together, our findings demonstrate that a combination of both, loss-of-function and gain-of-function mechanisms are likely to underlie the pathogenesis of SCA14, caused by mutations in the C1 domain of PKC $\mathrm{Y}$. Importantly, SCA14 patient iPSCs were found to accurately recapitulate pathological features observed in post-mortem SCA14 cerebellum, underscoring their potential as relevant disease models and their promise as future drug discovery tools.
\end{abstract}

Keywords: Ataxia, Stem cells, Purkinje cells, Neurodegeneration, Cerebellum, Protein kinase C gamma

\section{Introduction}

Spinocerebellar ataxia type 14 (SCA14) (OMIM 605361) most commonly represents with slowly progressive, relatively pure cerebellar ataxia characterized by gait disturbance, incoordination, mild dysarthria and nystagmus, with complex phenotypes such as myoclonus described in over a third of cases $[9,12]$. Brain MRI in SCA14 patients shows mild to severe cerebellar atrophy $[9,12]$, and loss of Purkinje cells has been described at post-mortem [7].

SCA14 is caused by mutations in the PRKCG gene encoding the conventional protein kinase $\mathrm{C}$ gamma (PKCY), which is particularly abundant in the Purkinje cells of the

\footnotetext{
* Correspondence: esther.becker@dpag.ox.ac.uk

${ }^{1}$ Department of Physiology, Anatomy and Genetics, University of Oxford, Sherrington Road, Oxford OX1 3PT, UK

Full list of author information is available at the end of the article
}

cerebellum [26]. To date, 40 mutations have been reported to cause SCA14 (Fig. 1a). Most of these mutations cluster in the regulatory $\mathrm{C} 1$ and $\mathrm{C} 2$ domains of $\mathrm{PKC} \gamma$ that respond to second messengers and control the activation and membrane translocation of PKC $\gamma$. Binding of calcium to the $\mathrm{C} 2$ domain initiates the activation of $\mathrm{PKC} Y$ and induces the rapid translocation of $\mathrm{PKC} \gamma$ from the cytoplasm to the plasma membrane, where it interacts with phospholipids. $\mathrm{PKC} \gamma$ is further allosterically activated by the binding of diacylglycerol (DAG) to the C1 domain, resulting in the release of a pseudo-inhibitory substrate that occupies the catalytic domain, and an open and active confirmation of $\mathrm{PKC} \gamma$ that allows phosphorylation of target substrates $[2,8]$.

The C1 domain is composed of two structurally and functionally similar cysteine-rich subdomains, $\mathrm{C} 1 \mathrm{~A}$ and $\mathrm{C} 1 \mathrm{~B}$, of which the latter is preferentially affected by SCA14 mutations (Fig. 1a). Despite the wealth of mutations identified in $\mathrm{PKC} \gamma$, the pathologic mechanisms underlying

(c) The Author(s). 2018 Open Access This article is distributed under the terms of the Creative Commons Attribution 4.0 International License (http://creativecommons.org/licenses/by/4.0/), which permits unrestricted use, distribution, and reproduction in any medium, provided you give appropriate credit to the original author(s) and the source, provide a link to the Creative Commons license, and indicate if changes were made. The Creative Commons Public Domain Dedication waiver (http://creativecommons.org/publicdomain/zero/1.0/) applies to the data made available in this article, unless otherwise stated. 


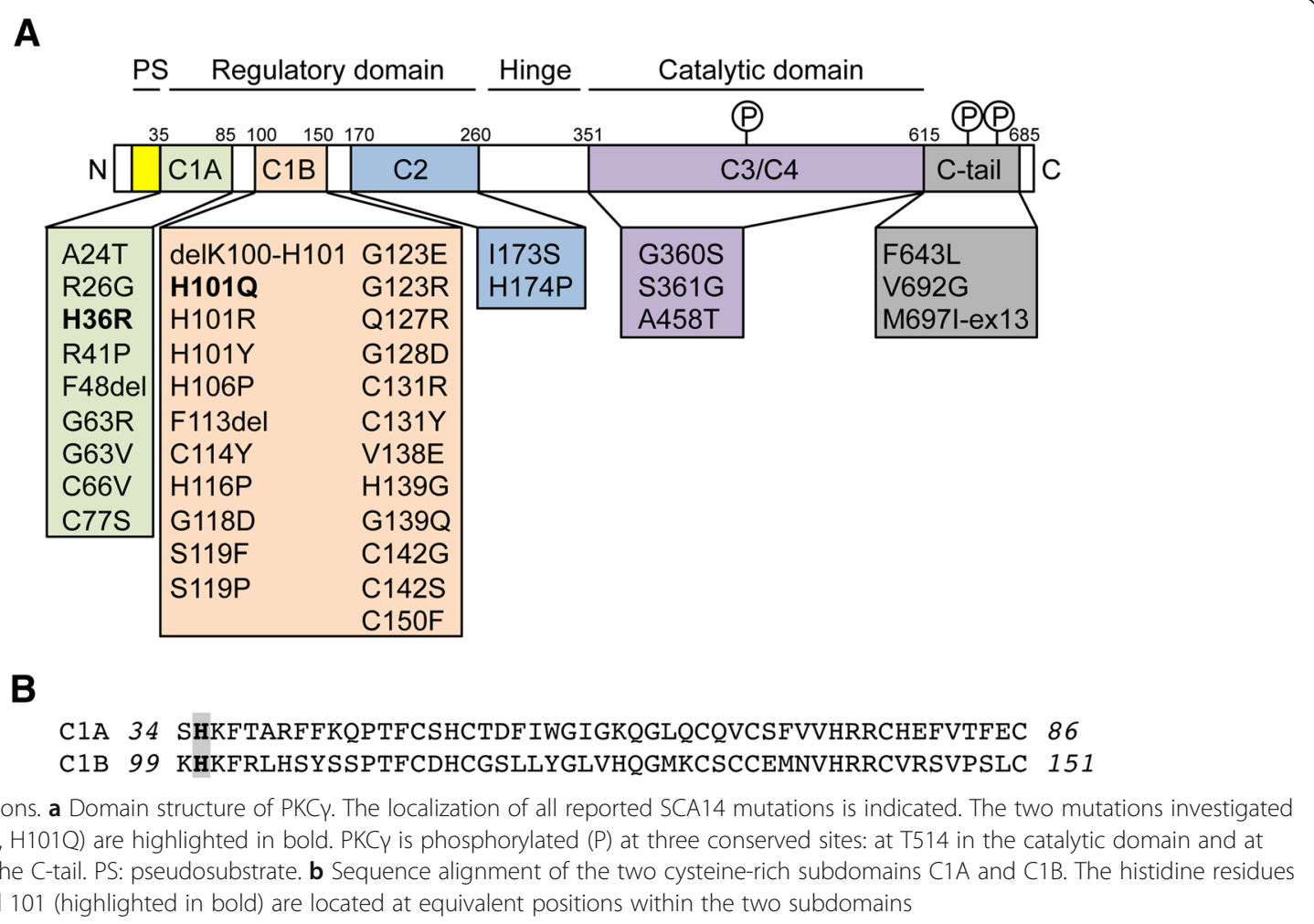

SCA14 remain unclear. Homozygous Prkcg knockout animals display only mild ataxia and show no loss of Purkinje cells [10, 22]. Therefore, the SCA14 phenotype is thought to result from a gain-of-function mechanism rather than haploinsufficiency. However, overexpression studies in cell lines and animals have yielded conflicting cellular disease mechanisms including increased kinase function [1, 43], impaired kinase function [42], protein aggregation [36] and impaired ubiquitin proteasome degradation [38], as well as aggregation-independent pathologies [37]. Thus, there is a need for authentic SCA14 models to better understand the underlying disease mechanisms.

Here, we have investigated the consequences of physiological expression of two SCA14 mutations in the C1 domain, H36R and H101Q, in both patient-derived induced pluripotent stem cells (iPSCs) and in SCA14 (H101Q) post-mortem cerebellum. We demonstrate that SCA14 patient iPSCs, in which PKC $\gamma$ is expressed at levels more likely to be relevant to normal physiology compared with previous in vitro models, recapitulate pathological features observed in post-mortem SCA14 cerebellum. We found that the SCA14 mutations result in a decrease of $\mathrm{PKC} \gamma$ at the plasma membrane upon activation, but increased PKC $\gamma$ aggregates in the cytoplasm of both patient iPSCs and Purkinje cells. We also observed lysosomal and autophagy impairment in SCA14 iPSCs and cerebellar tissue. $\mathrm{PKC} \gamma$ phosphorylation and downstream signaling were increased in the SCA14 iPSCs and cerebellum. Together, our findings suggest that SCA14 pathology is likely to be caused by the combination of a loss-of-function of PKCY at the plasma membrane and a gain-of-function of hyper-activated and mislocalized PKC $\gamma$.

\section{Materials and methods}

Generation and maintenance of iPSC lines

iPSC lines were derived from four SCA14 patients, two carrying an H36R and two carrying the H101Q mutation (Additional file 1: Figure S2). Reprogramming of donor fibroblasts to iPSCs was performed as described in the Additional file 1, and full characterization is provided in Additional file 1: Figure S2. iPSC lines from two age- and sex-matched healthy donors were used as controls (Additional file 1: Figure S2) and have been fully described elsewhere [16, 18]. iPSCs were maintained in feeder-free conditions on hESC-qualified Matrigel (Corning), in supplemented mTeSR (Stem Cell Technologies). Cells were passaged 1:3 every 4-5 days, using 0.5 mM EDTA (Invitrogen) [5]. For inhibitor experiments, iPSCs were treated with $400 \mathrm{nM}$ phorbol-12-myristate-13-acetate (PMA; R\&D Systems) or $200 \mathrm{nM}$ phorbol-12, 13-dibutyrate (PDBu) in PBS before harvesting.

\section{Quantitative real-time PCR}

Total RNA from iPSCs and cerebellar tissue was prepared using the RNeasy Mini Kit (Qiagen). RNA from 
human fetal cerebellar tissue was purchased (AMS Biotechnology (Europe) Ltd., Abingdon, UK). RNA was reverse transcribed to cDNA using the High-Capacity RNA-to-cDNA Kit (Applied Biosystems). Quantitative real-time PCR was performed using the Fast SYBR Green Master Mix (Applied Biosystems) on a StepOne Plus qPCR machine (Applied Biosystems). The relative PRKCG levels were quantified and normalized against the housekeeping gene $\beta$-actin with reference to a negative control, using standard DDCt techniques. Primers are listed in Additional file 1: Table S1.

\section{Biochemical assays}

Frozen tissue of human cerebellum was sampled from the inferior aspect, immediately lateral to the cerebellar tonsils (that is, adjacent to the areas with relative preservation of Purkinje cells on histology). Sampling sites were consistent between SCA14 and control cerebellum.

For the preparation of protein extracts, iPSCs were washed once with PBS and then lysed in cold Pierce ${ }^{\circ}$ RIPA buffer [25 mM Tris- $\mathrm{HCl} \mathrm{pH}=7.6,150 \mathrm{mM} \mathrm{NaCl}, 1 \%$ NP-40, $1 \%$ sodium deoxycholate, $0.1 \%$ sodium dodecyl sulphate (SDS)] (Thermo Fisher Scientific), supplemented with Complete Protease Inhibitor Cocktail (Roche) and PhosSTOP Phosphatase Inhibitor Cocktail (Roche). Protein lysates were incubated on ice for $10 \mathrm{~min}$ and subsequently centrifuged at $14,000 \mathrm{~g}$ for $20 \mathrm{~min}$ at $4{ }^{\circ} \mathrm{C}$. Snap-frozen human cerebellar tissue was homogenized in cold RIPA buffer, followed by 30 -s sonication. The cerebellar lysate was incubated on ice for $10 \mathrm{~min}$ before centrifugation at $14,000 \mathrm{~g}$ for $30 \mathrm{~min}$ at $4{ }^{\circ} \mathrm{C} .50 \mu \mathrm{g}$ of protein extracts were analyzed by SDS-PAGE and immunoblotting.

For the preparation of (in)soluble fractions, snap-frozen cerebellar tissue was homogenized in cold lysis buffer [ $1 \%$ Triton X-100, $20 \mathrm{mM}$ Tris, $\mathrm{pH}=7.5,5 \mathrm{mM}$ ethylene glycol-bis( $\beta$-aminoethyl ether)-N,N, $\mathrm{N}^{\prime}, \mathrm{N}^{\prime}$-tetraacetic acid (EGTA), $150 \mathrm{mM} \mathrm{NaCl}$, Complete Protease Inhibitor Cocktail, PhosSTOP Phosphatase Inhibitor Cocktail], followed by a 10-min incubation on ice and centrifugation at $14,000 \mathrm{~g}$ for $30 \mathrm{~min}$ at $4{ }^{\circ} \mathrm{C}$. The supernatant was collected as Triton-soluble fraction. The pellet was re-suspended in cold Pierce ${ }^{\circ}$ RIPA buffer (Triton-insoluble fraction) and sonicated for 10-20 s. Equal volumes of soluble and insoluble fractions were loaded for SDS-PAGE and analyzed by immunoblotting.

A list of primary and secondary antibodies can be found in Additional file 1: Table S2. Antibody binding was detected by enhanced chemoluminescence (ECL, GE Healthcare). The intensity of bands was quantified using ImageJ software (NIH). Data were normalized to Actin levels and respective control bands and analyzed using GraphPad Prism 7 (GraphPad Software, Inc.). All data are represented as the mean of three independent experiments \pm SEM. Statistical significance was assessed by ANOVA with Bonferroni's post-hoc test, with $p<0.05$ considered statistically significant.

\section{Immunostaining}

iPSCs were fixed in $4 \%$ paraformaldehyde at room temperature for $20 \mathrm{~min}$ or in ice-cold methanol at $-20{ }^{\circ} \mathrm{C}$ for $15 \mathrm{~min}$. Fixed cells were washed three times with PBS for $5 \mathrm{~min}$ and subjected to immunostaining as previously described [44]. A list of primary and secondary antibodies can be found in Additional file 1: Table S2. Images were analysed using ImageJ. The size of aggregates was measured using the ImageJ Cell Counter plugin. The area of signals was measured using Threshold and Area Measurement. The co-localization of labelled proteins was quantified using Just Another Colocalization Plugin (JACoP) [6].

Immunohistochemistry of human cerebellar sections was performed as follows. 5- $\mu \mathrm{m}$ sections were cut from formalin-fixed paraffin-embedded blocks from the vermis, paravermis and lateral neocerebellum, including dentate nucleus. The index case was matched to two control cases. All three cases were assessed for $\mathrm{PKC} \gamma$ reactivity outside the cerebellum and screened for age-related neurodegenerative pathology. De-identified sections were de-waxed through xylene, and rehydrated through decreasing concentrations of alcohol before being pre-treated for $30 \mathrm{~min}$ in $10 \%$ concentrated $(30 \%) \mathrm{H}_{2} \mathrm{O}_{2}$ and distilled water to block endogenous peroxidase. Heat-induced epitope-retrieval was performed using autoclave boiling at $121{ }^{\circ} \mathrm{C}$ for $10 \mathrm{~min}$. Sections were then rinsed with Tris-buffered saline and blocked with normal goat serum (1:10 in TBS-T) for $30 \mathrm{~min}$. Primary antibodies (Additional file 1: Table S2) were incubated overnight at $4{ }^{\circ} \mathrm{C}$ and visualized using the Dako Envision+ kit and HRP-DAB signal (Agilent). Positive and negative controls were used for each antibody. No staining was seen when the primary antibody was omitted. Sections were viewed and photographed with an Olympus BX43 microscope and Olympus cellSense software.

\section{Results \\ Cerebellar pathology in SCA14}

Given the multitude of, often conflicting, phenotypes reported in the literature that might be caused by mutations in $\mathrm{PKC} Y$ in heterologous models, we set out to investigate the pathological changes in SCA14 in patient-derived cells and post-mortem cerebellum. We focused on two different SCA14 mutations, H36R and H101Q. Both of these mutations are located at equivalent positions in the $\mathrm{C} 1 \mathrm{~A}$ and $\mathrm{C} 1 \mathrm{~B}$ subdomains of the regulatory domain of $\mathrm{PKC} \gamma$ (Fig. 1) [32] and are implicated in zinc coordination and phorbol ester binding [11].

Affected individuals had slowly progressive adult onset ataxia typical of SCA14, with moderate gait ataxia, mild 
dysarthria, titubation and relatively mild nystagmus. The H101Q family had pure ataxia, without additional features described in other pedigrees such as myoclonus and seizures. MRI showed moderate to severe generalized atrophy of the cerebellum (Fig. 2a). One individual from the SCA14 H101Q family underwent autopsy when he died of 'natural causes' at the age of 90 years (Additional file 1: Figure S1). Brain tissue was examined according to standard protocols for neurodegenerative disease, which included screening for Alzheimer disease, Lewy body disease and TDP-43 proteinopathy. We found Braak II/III neurofibrillary Alzheimer type pathology and mild cerebrovascular disease. No Lewy body or TDP-43 proteinopathy was identified. We used a sequestosome1/p62 antibody as a highly sensitive screening tool for generic protein aggregates. We did not find any neuropathology that could not be explained by Alzheimer-related changes.

To identify Purkinje cells, tissue sections were immunolabelled with an antibody against the calcium-binding protein Calbindin D-28 k. We observed severe loss (estimated to be $80 \%$ ) of Purkinje cells in all lobules of the neocerebellum, associated with Bergmann gliosis. However, Purkinje cells in the cerebellar tonsils and adjacent flocculonodular lobe were relatively preserved (Fig. 2b). Neurons of the deep cerebellar nuclei, pons

A

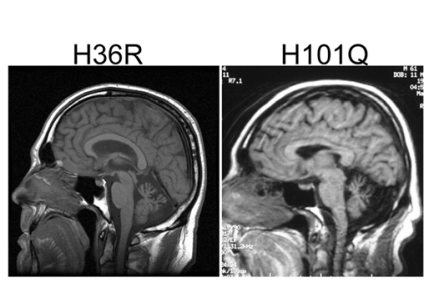

B

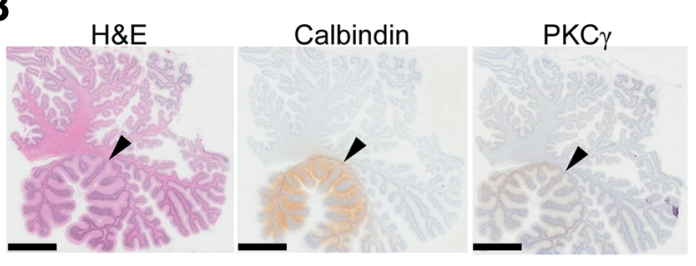

C

D
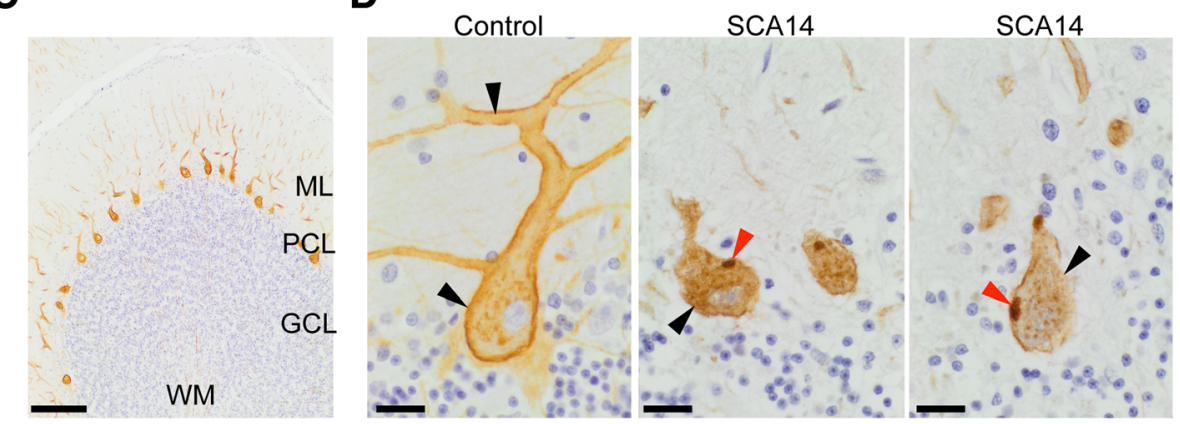

E

$\mathbf{F}$
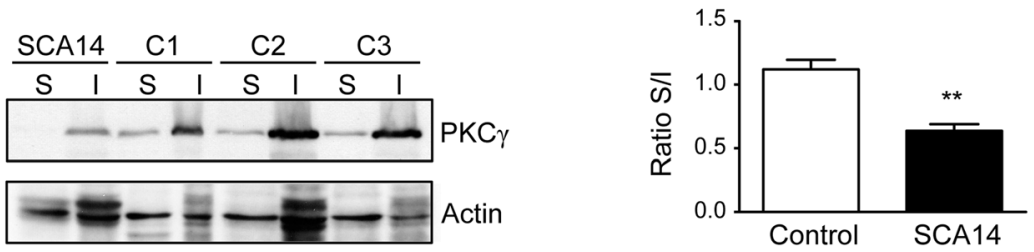

Fig. 2 Cerebellar pathology in SCA14. a Brain MRI imaging of SCA14 patients carrying the H36R and H101Q mutations, respectively, shows marked cerebellar atrophy. $\mathbf{b}$ Neurodegeneration of SCA14 cerebellum. There is severe loss of Purkinje cells from the lateral neocerebellum. Purkinje cells in the tonsil (and flocconodular lobe) are relatively preserved (black arrowheads). Brain sections were stained with hematoxylin and eosin (H\&E) (left panel), and with antibodies against Calbindin-28 k (centre) and PKCY (right panel). Scale bar: 5 mm. c Normal PKCY pattern from an age-matched control cerebellum. ML: molecular layer, PCL: Purkinje cell layer, GCL: granule cell layer, WM: white matter. Scale bar: 200 m. d PKCY staining of control (left) and SCA14 cerebellum (centre and right panels). In control cerebellum, PKCY showed distinct expression at the plasma membrane, both around the soma and primary and secondary dendrites (black arrowheads), with minor granular staining in the perinuclear cytoplasm. In SCA14 cerebellum, homogeneous circumferential plasmalemma localization was lost (black arrowheads) and large cytoplasmic PKCY aggregates were found, some apparently still linked to fragments of plasma membrane (red arrowheads). Scale bar: $20 \mu \mathrm{m}$. e, $\mathbf{f}$ Enrichment of mutant PKCY in the Triton-X-100-insoluble fraction in SCA14 cerebellum compared to controls. Cerebellar tissue lysates were separated into Triton-X-100-soluble (S) and -insoluble (I) fractions. Equal volumes of soluble and insoluble fractions were loaded for SDS-PAGE and analyzed by immunoblotting for PKCY. (e). Actin: loading control. The intensity of the bands was quantified and the level of PKC $y$ was normalized against the loading control. The ratio of normalized PKCY present in the soluble versus insoluble fractions (Ratio S/I) is shown $\left(n=3,{ }^{* *} p<0.01\right.$, unpaired students' t-test). (f). S: Triton-X-100-soluble fraction, I: Triton-X-100-insoluble fraction 
and inferior olive were not obviously depleted. Thus, we conclude that SCA14 seems to be a pure Purkinje cell neuronopathy, predominantly affecting the lateral parts of the cerebellar hemispheres (neocerebellum). This is consistent with the highly restricted expression pattern of $\mathrm{PKC} Y$ in human control cerebellum (Fig. 2c). No other cerebellar cell type expressed $\mathrm{PKC} \gamma$. The remaining Purkinje cells displayed variable degrees of dendritic and somatic atrophy compared to control tissue (Fig. 2d). In age-matched control autopsy material, $\mathrm{PKC} \gamma$ was localized to the plasma membrane and cytoplasmic puncta in the soma and primary dendrite of Purkinje cells (Fig. 2d). This staining pattern is consistent with the localization of $\mathrm{PKC} Y$ in rodent Purkinje cells $[26,39]$. In contrast, $\mathrm{PKC} \gamma$ staining at the plasma membrane was lost in SCA14 Purkinje cells and associated with large cytoplasmic aggregates in the soma, sometimes preserving a link to the plasma membrane (Fig. 2d). Loss of PKCY staining was particularly pronounced in the dendrites. PKC $\gamma$ aggregates were unique to Purkinje cells. Compared to Purkinje cells, only minimal expression of $\mathrm{PKC} \gamma$ is seen in any other part of the adult human brain. In our hands, the only extracerebellar region with faint expression in age-matched controls corresponded to the CA1-CA4 sectors of the hippocampus. However, unlike in the cerebellum, staining revealed only diffuse neuropil positivity, and no distinct membrane, soma, dendrite or axonal neuronal expression (data not shown). The SCA14 index case showed no aggregates or other morphological $\mathrm{PKC} \gamma$ abnormalities in the hippocampal formation compared with controls. We conclude from our immunohistochemical studies that cytoplasmic and membrane expression of $\mathrm{PKC} \gamma$ in adult cerebellar Purkinje cells is several orders of magnitude higher than in any other cell type of the human brain. We postulate that this underpins selective vulnerability and thus clinical presentation, and that loss of PKCY cell membrane binding, cytoplasmic aggregation and Purkinje cell death represent the morphological substrate of the SCA14 H101Q mutation in human brain.

Many neurodegenerative diseases are characterized by the formation of disease-specific inclusions including Parkinson's Disease, Huntington's Disease and the polyglutamine SCAs $[25,35]$. Inclusion bodies are generated by aggregation of misfolded proteins and often become detergent-insoluble. To formally confirm the insolubility of the PKCY aggregates in SCA14 cerebellum, we carried out biochemical fractionation of cerebellar tissue into Triton X-100-soluble and -insoluble fractions. $\mathrm{PKC} Y$ was found in both soluble and insoluble fractions in control cerebellum (Fig. 2e). In contrast, $\mathrm{PKC} \gamma$ in SCA14 cerebellum was found almost exclusively in the insoluble fraction (Fig. 2e, f). Together, these findings suggest that in SCA14 Purkinje cells, $\mathrm{PKC} \gamma$ is mislocalized and aggregated in detergent-insoluble inclusions.

\section{Generation of SCA14 human iPSCs}

To better understand the pathological mechanisms that cause SCA14, we generated human iPSC lines from fibroblasts obtained from two patients carrying the H36R mutation and from two patients with the H101Q mutation (Additional file 1: Figure S1 \& S2, Suppl. Methods). At least two iPSC clones were generated from each patient. Ageand sex-matched control iPSC lines, reprogrammed using Sendai reprogramming viruses in the same laboratory, generated through the Oxford Parkinson's Disease Centre, have been published previously $[16,18]$. All iPSC lines displayed embryonic stem cell-like morphology and expressed the pluripotency-associated proteins Tra-1-60 and Nanog (Additional file 1: Figure S2B \& D). Clearance of viral transgenes was confirmed by qRT-PCR (Additional file 1: Figure S2C). Genome integrity was confirmed by Illumina SNP arrays (Additional file 1: Figure S2E). PRKCG genotypes were confirmed in all quality-checked iPSC lines by Sanger sequencing (Additional file 1: Figure S2F).

SCA14 mutations cause PKC $\gamma$ aggregation in human iPSCs Although $\mathrm{PKC} \gamma$ is generally known to be a neuron-specific kinase, we identified robust expression of PRKCG RNA in both control and patient iPSCs human iPSCs (Fig. 3a, b), consistent with previous reports [24]. This prompted us to investigate the cellular phenotypes of iPSCs expressing mutant PKC $\gamma$. Similar to our observations in post-mortem cerebellar tissue, wildtype $\mathrm{PKC} \gamma$ was present in small cytoplasmic puncta, which partially co-localized with the cis-Golgi marker GM130, early endosomal marker EEA1 and recycling endosomal marker RAB11 (data not shown). In contrast, mutant $\mathrm{PKC} \gamma$ formed large aggregates in the cytoplasm (Fig. 3c, d), with little co-localization with Golgi and endosomal markers (data not shown). This staining pattern was observed for both SCA14 mutations, H36R and $\mathrm{H} 101 \mathrm{Q}$.

Prolonged activation of PKC results in its accumulation in the detergent-insoluble fraction, where it is subjected to dephosphorylation and degradation $[2,15,33]$. To address whether activation of mutant $\mathrm{PKC} \gamma$ further enhanced its aggregation, we treated control and SCA14 iPSCs with $400 \mathrm{nM}$ of phorbol 12-myristate 13-acetate (PMA), a potent PKC activator. Stimulation with PMA led to a more significant increase in the size of aggregates in SCA14 patient cells compared to controls (Fig. 3e). DMSO vehicle control did not affect $\mathrm{PKC} \gamma$ aggregation (Additional file 1: Figure S3). Together, these results indicate that the SCA14 H36R and H101Q mutations cause the aggregation of $\mathrm{PKC} \gamma$ in the cytoplasm of iPSCs, which is further enhanced following $\mathrm{PKC} \gamma$ activation.

\section{Reduced membrane targeting of mutant PKC $\gamma$}

The $\mathrm{C} 1$ domain mediates binding of $\mathrm{PKC} Y$ to DAG and phospholipids at the plasma membrane [8]. As both 
A

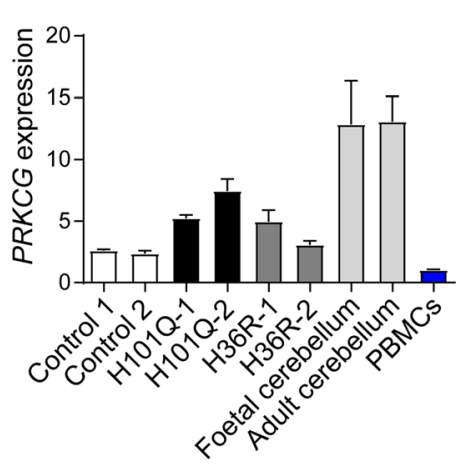

C

$\mathrm{PKC} \gamma /$ peptide
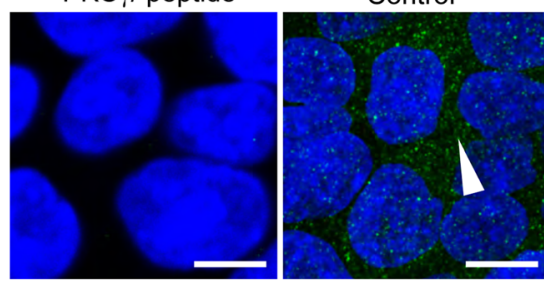

SCA14 (H101Q)

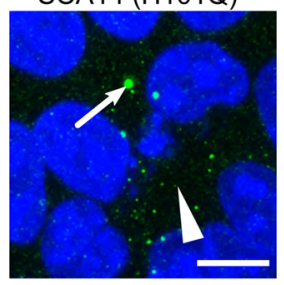

SCA14 (H36R)
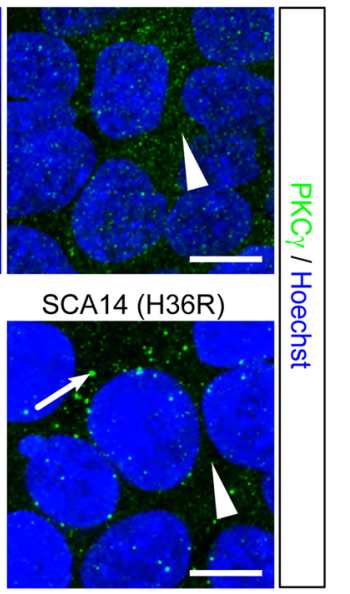

E

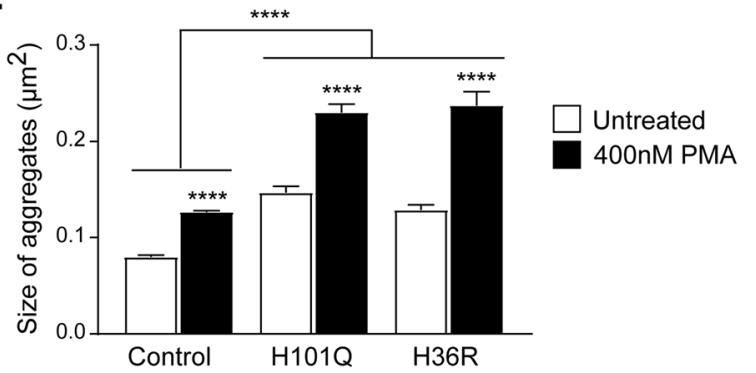

B

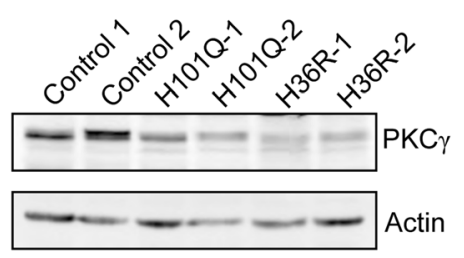

D

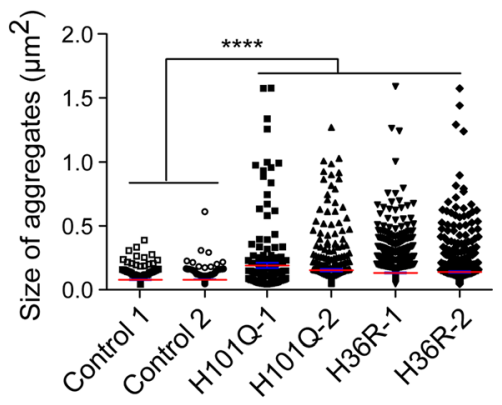

Fig. 3 Mutant PKC forms cytoplasmic aggregates in iPSCs. a PRKCG mRNA expression in control and patient iPSC lines. RNA extracted from fetal and adult human cerebellum was included as positive controls. PRKCG is not expressed in peripheral blood mononuclear cells (PBMCs) according to data from GTEx, BioGPS, and CGAP SAGE, and thus, RNA extracted from PBMCs was used as negative control. PRKCG gene expression levels were normalized to housekeeping gene $\beta$-actin, and are shown relative to negative control. $\mathbf{b}$ PKCY protein expression in control and patient iPSC lines. Actin: loading control. c Immunostaining of iPSC lines for PKCY. Specificity of the anti-PKCY antibody was confirmed by peptide absorption assay (top left panel). Small punctate staining of PKCY (white solid arrowheads) was observed in the cytoplasm of control iPSCs and SCA14 iPSCs, while large cytoplasmic aggregates (white arrows) were only present in SCA14 iPSCs. Cell nuclei are visualized by Hoechst staining. Scale bar: $10 \mu \mathrm{m}$. d PKC formed significantly larger aggregates in SCA14 iPSCs compared to control iPSCs $\left(n=3,{ }^{* * *} p<0.0001\right.$, ANOVA followed by Bonferroni's post-hoc test). e Treatment with $400 \mathrm{nM}$ PMA, a potent PKC $Y$ activator, both wildtype and mutant PKC $\mathrm{yggregates}$ increased in size. Compared to control, PKCy formed significantly larger aggregates in SCA14 iPSCs following PMA treatment $\left(n=3,{ }^{* * * *} p<0.0001\right.$, two-way ANOVA followed by Bonferroni's post-hoc test)

SCA14 mutations investigated in this study are located in the $\mathrm{C} 1$ domain, we next determined whether SCA14 mutants would be impaired in their membrane targeting. Interestingly, as described above, $\mathrm{PKC} \gamma$ immunostaining at the plasma membrane of Purkinje cells was markedly reduced in SCA14 post-mortem cerebellar tissue (Fig. 2d). To test whether membrane translocation of $\mathrm{PKC} \gamma$ was affected in patient iPSCs, cells were treated with $400 \mathrm{nM}$ 
PMA to activate PKCy. In control iPSCs, PKCy colocalization with sodium potassium ATPase at the plasma membrane increased after 5 min of PMA treatment, and after 15 min of PMA treatment, $\mathrm{PKC} \gamma$ was found again in the cytoplasm (Fig. 4a, b). In contrast, mutant $\mathrm{PKC} \gamma$ remained aggregated in the cytoplasm and did not translocate to the plasma membrane in response to PMA treatment (Fig. 4a, b). Similar results were obtained following treatment with phorbol 12,13-dibutyrate

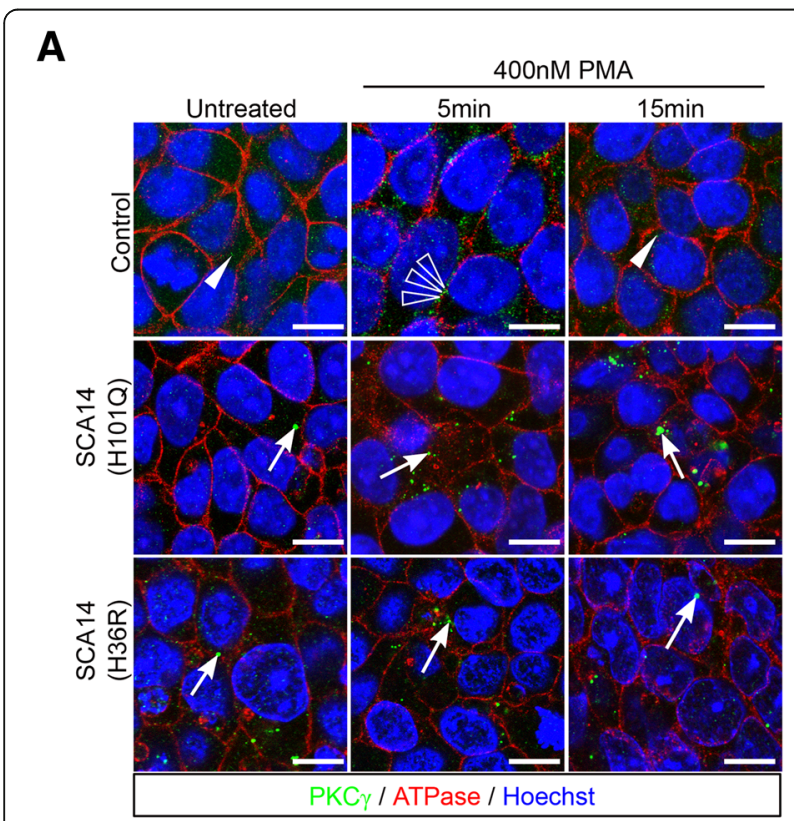

B

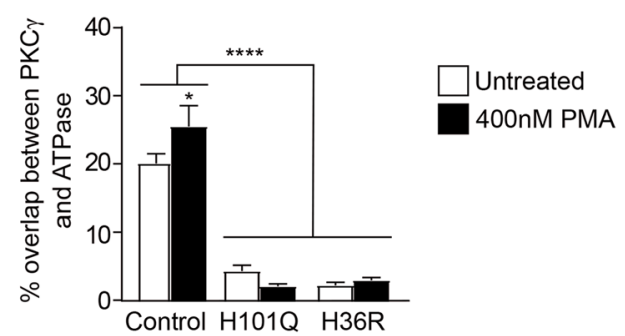

Fig. 4 SCA14 mutations reduce PMA-induced membrane translocation of PKC $\gamma$. a Control and patient iPSCs were immunostained for PKCY before or after treatment with PMA. The cell membrane was stained with an antibody against sodium potassium ATPase. Cell nuclei are visualized by Hoechst staining. In unstimulated control iPSCs, PKC $y$ was expressed as small dots in the cytoplasm (white solid arrowhead). After 5 min of PMA treatment, PKC $Y$ was found at the plasma membrane (white hollow arrowheads), and returned to the cytoplasm after 15 min of PMA treatment (white solid arrowhead). In unstimulated SCA14 iPSCs, large aggregates (white arrowheads) of PKC $y$ were present in the cytoplasm. PKCY inclusions remained in the cytoplasm (white arrowheads) throughout the treatment with PMA. Scale bar: $10 \mu \mathrm{m}$. b PKCY in SCA14 iPSCs showed significantly less membrane association than in control iPSCs in response to PMA stimulation $\left(n=3,{ }^{* * * *} p<0.0001\right.$, two-way ANOVA followed by Bonferroni's post-hoc test)
$(\mathrm{PDBu})$, an alternative $\mathrm{PKC} \gamma$-activating phorbol ester (Additional file 1: Figure S4A). Together, these findings indicate that mutant $\mathrm{PKC} \gamma$ is impaired in its ability to translocate to, or be retained at, the plasma membrane.

\section{Impaired degradation of SCA14 PKC $\gamma$ aggregates}

We next investigated the cellular responses to mutant and aggregated PKC $\mathrm{P}$. Cells operate two major protein degradation machineries: the ubiquitin proteasome system (UPS) and autophagy [14]. Impairment of both the UPS and autophagy have been associated with neurodegenerative disorders $[14,31]$. This is further supported by the accumulation of intraneuronal aggregates of misfolded proteins in many neurodegenerative disorders [25, 31, 35]. Most of these aggregates are visible with light microscopy with immunohistochemistry against the disease-defining protein species (e.g. alpha-synuclein, C-terminal huntingtin) and components of the ubiquitin proteasome or macroautophagy systems. Interestingly, we did not find co-localization of the $\mathrm{PKC} \gamma$ aggregates with antibodies to ubiquitin or p62 in SCA14 cerebellum (data not shown). We next assessed whether mutant $\mathrm{PKC} \gamma$ aggregates in iPSCs were tagged with ubiquitin in an attempt by the cells to clear the aggregates. Control and patient iPSCs were immunostained with antibodies against $\mathrm{PKC} \gamma$ or ubiquitin in the presence or absence of PMA or PDBu. No ubiquitin-positive $\mathrm{PKC} \gamma$ aggregates were identified (Additional file 1: Figure S4), consistent with the results obtained in post-mortem SCA14 cerebellum.

The absence of PKCY ubiquitination led us to investigate whether mutant PKCY aggregates might be degraded through a different cellular pathway. We first looked at the formation of autophagosomes using immunostaining microtubule-associated protein 1 light chain 3 (LC3), a central protein in the autophagy pathway. In control iPSCs, we observed a significant increase in the overlap between PKCY and LC3 following activation by PMA or PDBu (Fig. 5a, b; Additional file 1: Figure S4). In contrast, there was already a significant overlap between SCA14 PKCY and LC3 in unstimulated iPSCs (Fig. 5a, b). This overlap did not increase upon further PKCY activation by PMA or PDBu (Fig. 5a, b; Additional file 1: Figure S4), despite the increased formation of $\mathrm{PKC} Y$ aggregates observed (Fig. 3e). Overall, autophagosome levels did not significantly change in the presence of mutant $\mathrm{PKC} \gamma$ aggregates or upon $\mathrm{PKC} \gamma$ activation (Fig. $5 c, d$ ). These results indicate that aggregated mutant $\mathrm{PKC} Y$ is not cleared efficiently by the autophagosome in SCA14 iPSCs.

Aggregated proteins that are engulfed by autophagosomes are subsequently degraded through the fusion with lysosomes [31]. Alternatively, aggregated proteins can also be degraded by lysosomes through autophagosome-independent pathways [14]. We found that a small 
A

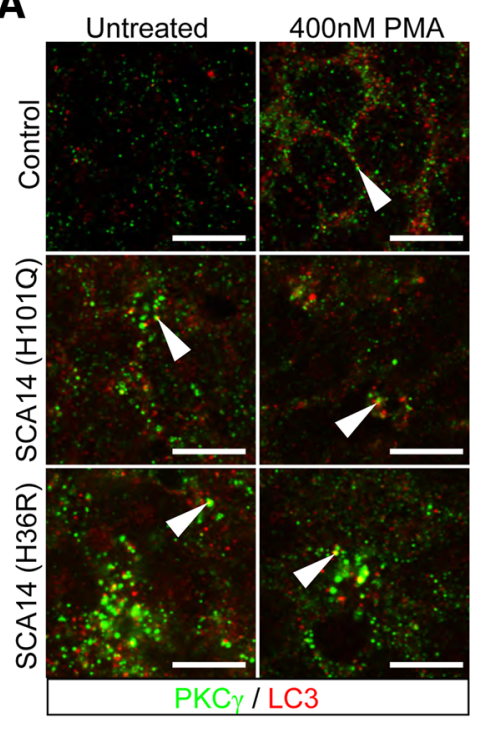

E

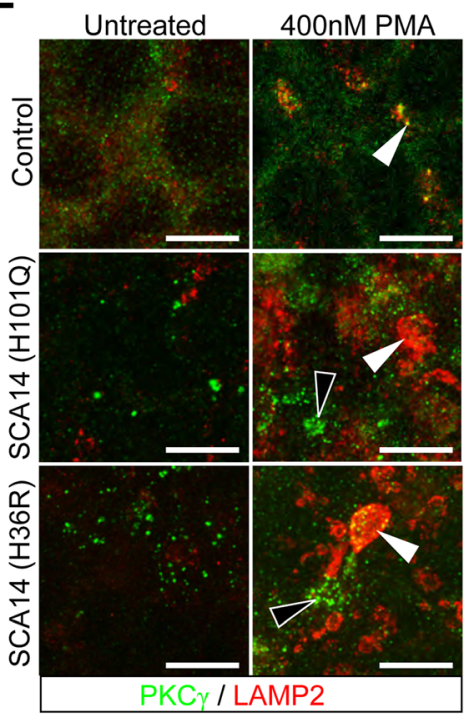

B

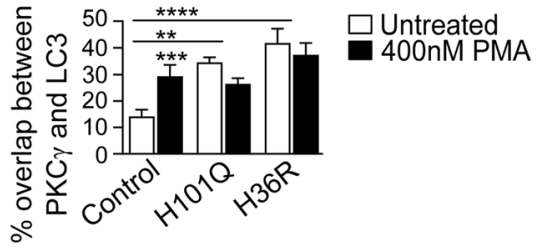

C

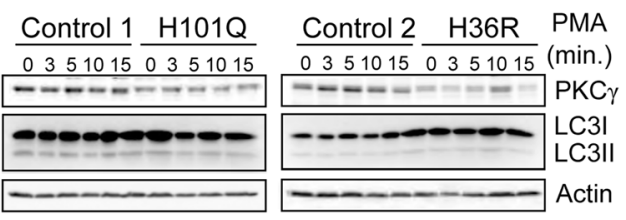

D

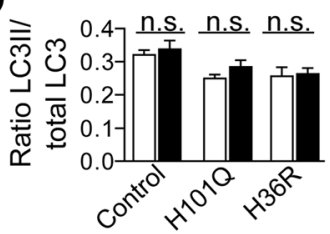

$F$

G
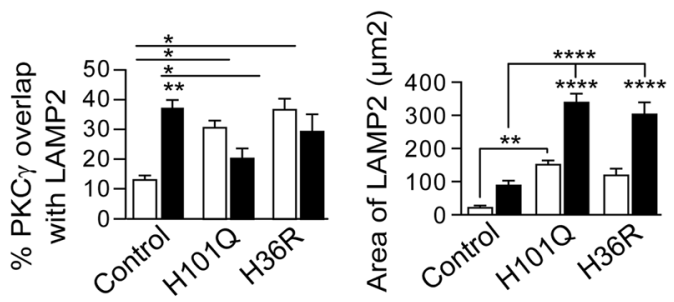

H

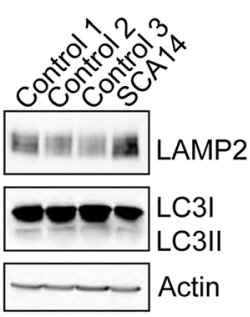

Fig. 5 Impaired degradation of SCA14 PKCY aggregates. a, b Control and patient iPSCs were immunostained for PKC $y$ and the autophagosomal marker LC3 before or after treatment with PMA for 15 min. In control iPSCs, PKCY co-localization with LC3 (white solid arrowheads) increased upon treatment with PMA. In untreated SCA14 iPSCs, there was already a significant overlap with LC3 (white solid arrowheads), which did not further increase upon PKC $y$ activation $\left(n=3,{ }^{* *} p<0.01,{ }^{* * *} p<0.001\right.$, ${ }^{* * * *} p<0.0001$, two-way ANOVA followed by Bonferroni's post-hoc test). c Lysates of iPSCs were subjected to immunoblotting for PKCY and LC3. LC3I represents free cytosolic cleaved LC3. LC3II represents LC3 that is anchored to the autophagosome membrane and indicates autophagosome load. $\mathbf{d}$ Ratio of LC3II/total LC3 levels remained constant in control and SCA14 iPSCs following PMA treatment. e, $\mathbf{f}$ Control and patient IPSCs were immunostained for PKCY and the lysosomal marker LAMP2 before or after treatment with PMA for 15 min. In control iPSCs, co-localization of PKC $y$ with LAMP2 increased upon activation (white solid arrowheads). In SCA14 iPSCs, by contrast, lysosomes fused together into larger vesicles enclosing PKCY aggregates (white arrowheads) in the presence of PMA. However, the majority of PKC $y$ aggregates did not co-localize with LAMP2-postive lysosomes (white hollow arrowheads) $\left(n=3,{ }^{*} p<0.05\right.$, ${ }^{*} p<0.001$, two-way ANOVA followed by Bonferroni's post-hoc test). $\mathbf{g}$ The area of LAMP2 signal, representing the formation of lysosomes, significantly increased in both control and SCA14 iPSCs following PMA treatment. The lysosomal area was significantly larger in SCA14 iPSCs compared to control iPSCs $\left(n=3,{ }^{* *} p<0.01,{ }^{* * * *} p<0.0001\right.$, two-way ANOVA followed by Bonferroni's post-hoc test). $\mathbf{h}$ Cerebellar lysates were subjected to immunoblotting for LAMP2 and LC3. LC3I represents free cytosolic cleaved LC3. LC3II represents LC3 that is anchored to the autophagosome membrane and indicates autophagosome load 
proportion of wildtype $\mathrm{PKC} \gamma$ co-localized with the lysosomal marker LAMP2 (lysosome-associated membrane protein 2) in the absence of PMA treatment (Fig. 5e, f). Following $\mathrm{PKC} \gamma$ activation in control iPSCs, both the lysosomal area, and the co-localization of $\mathrm{PKC} \gamma$ and LAMP2 significantly increased (Fig. 5f, g; Additional file 1: Figure S4). In SCA14 iPSCs, a significant enlargement of the lysosomal compartment and co-localization of $\mathrm{PKC} Y$ with LAMP2 was already observed prior to PMA treatment. (Fig. 5e-g). Upon PMA treatment, lysosomes fused together and formed very large vesicles (Fig. 5e, g; Additional file 1: Figure S4). Some PKCY aggregates were found to be enclosed within these large lysosomes. However, the majority of mutant $\mathrm{PKC} Y$ did not co-localize with LAMP2 (Fig. 5e, f). Moreover, compared to control iPSCs, less $\mathrm{PKC} \gamma$ was found to co-localize with LAMP2 following activation in SCA14 iPSCs (Fig. 5f). Together, these results suggest that despite lysosomal enlargement, aggregated mutant $\mathrm{PKC} Y$ is not efficiently targeted by lysosomes and thus accumulates as cytosolic aggregates in SCA14 iPSCs. Interestingly, increased expression of LAMP2 but no change in LC3 levels were also found in SCA14 cerebellum (Fig. 5h) indicating that the findings in SCA14 iPSCs reflect cerebellar pathology.

\section{Increased PKC $\gamma$ kinase activity in SCA14 patient cells}

Phosphorylation is known to play an important role in regulating $\mathrm{PKC} \gamma$, rendering $\mathrm{PKC} \gamma$ in a catalytically competent conformation, and protecting it from degradation [2]. $\mathrm{PKC} Y$ phosphorylation occurs sequentially at three conserved residues: phosphoinositide-dependent kinase 1 (PDK1) phosphorylates PKCY within the activation loop (T514), and autophosphorylation occurs within the turn motif (T655) and the hydrophobic motif (T674) at the C-terminal tail (Fig. 1a). Having identified that mutant $\mathrm{PKC} \gamma$ is not efficiently cleared in SCA14 patient cells, we next determined whether its phosphorylation status might be altered compared to wildtype PKC $\gamma$, which might affect its stability and kinase activity. Although overall $\mathrm{PKC} \gamma$ expression was lower in SCA14 iPSCs than control cells (Figs. 4b \& 6a), PKCY was highly phosphorylated at T514 and T674 in SCA14 iPSCs as determined by immunoblotting with phospho-specific antibodies (Fig. 6a, b). We also analyzed the phosphorylation status of PKCY in SCA14 (H101Q) cerebellar tissue. Less $\mathrm{PKC} \gamma$ protein was present in SCA14 cerebellum compared to controls (Fig. 6c). We noted a similar reduction in Calbindin protein levels, consistent with the loss of Purkinje cells in the SCA14 cerebellum that was observed histopathologically (Fig. 2b). Despite the reduction in total $\mathrm{PKC} Y$ protein level, there was no reduction in phosphorylation levels of the $\mathrm{PKC} Y$ activation loop (Fig. 6c). Quantification of the phosphoT514$\mathrm{PKC} Y$ levels in three independent experiments showed that net phosphorylation of $\mathrm{PKC} \gamma$ was significantly increased in
SCA14 cerebellum compared to control tissue (Fig. 6d). Together with previous results, these findings indicate that the SCA14 mutations H36R and H101Q promote the aberrant maturation of $\mathrm{PKC} \gamma$ into a catalytically competent and stable conformation.

Phosphorylated $\mathrm{PKC} \gamma$ increases its affinity for $\mathrm{Ca}^{2+}$ and promotes substrate binding $[2,8]$. We therefore next asked whether the SCA14 mutations affect downstream $\mathrm{PKC} Y$ signaling and assessed the phosphorylation status of several $\mathrm{PKC} \gamma$ substrates. First, we employed a pan-phospho-PKC substrate antibody that recognizes cellular proteins (Ser-)phosphorylated at PKC consensus motifs. PKC substrate phosphorylation was consistently higher in iPSCs derived from SCA14 patients than in controls (Fig. 6a). Moreover, we detected a robust increase in PKC substrate phosphorylation in the SCA14 cerebellum compared to controls (Fig. 6e). We also assessed the phosphorylation status of a well-known PKC target in the brain, myristoylated alanine-rich C-kinase substrate (MARCKS). Using a phospho-specific antibody, we detected elevated phospho-MARCKS levels in the SCA14 cerebellum compared to controls (Fig. 6e). Together, these findings suggest that the SCA14 mutations H36R and $\mathrm{H} 101 \mathrm{Q}$ cause increased kinase activity of $\mathrm{PKC} \gamma$ in both patient iPSCs and cerebellum.

\section{Discussion}

In this study, we provide novel insights into the pathogenesis of SCA14. We present a unique in vitro model using human patient-derived iPSCs carrying two distinct SCA14 mutations in the $\mathrm{C} 1$ domain of PKC $\gamma, \mathrm{H} 36 \mathrm{R}$ and H101Q, respectively, that recapitulate key pathological findings observed in SCA14 cerebellum. Our findings indicate that SCA14 is likely to be caused by three interconnected pathogenic mechanisms (Fig. 7): (i) SCA14 mutations in the $\mathrm{C} 1$ domain enhance the aggregation of $\mathrm{PKC} \gamma$, aided by insufficient protein degradation, (ii) a reduction of mutant $\mathrm{PKC} \gamma$ at the plasma membrane is likely to decrease its interaction with target substrates, and (iii) extended cytoplasmic retention of hyper-active $\mathrm{PKC} Y$ results in aberrant phosphorylation of substrates in the cytoplasm.

This study sheds light on the important question of how $\mathrm{PKC} Y$ harboring mutations in the $\mathrm{C} 1$ domain causes SCA14 pathology. Mutations in other domains of PKCY might cause disease through other or additional mechanisms. Indeed, other $\mathrm{PKC} \gamma$ mutations in overexpression studies have been reported to drive a plethora of cellular phenotypes, which often contradict each other [1, 43]. This is the first study that investigates the consequences of more physiological levels of expression of two distinct SCA14 mutations in relevant human models, iPSCs and post-mortem cerebellar tissue. Only one postmortem brain of a SCA14 patient with a H101Y mutation has 
A

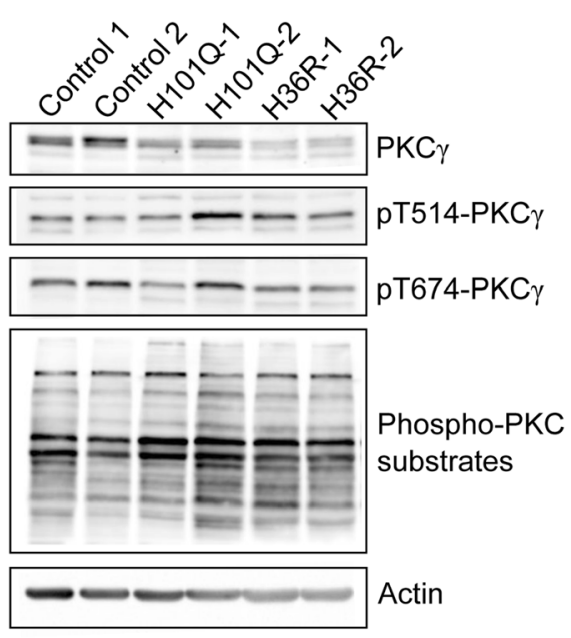

C

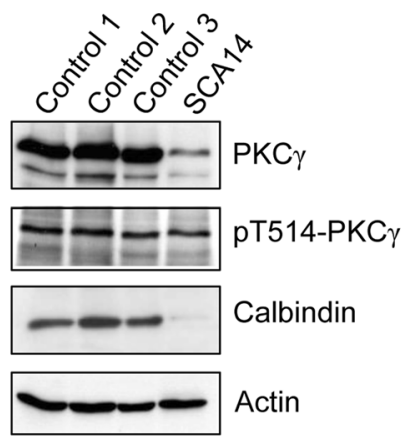

E

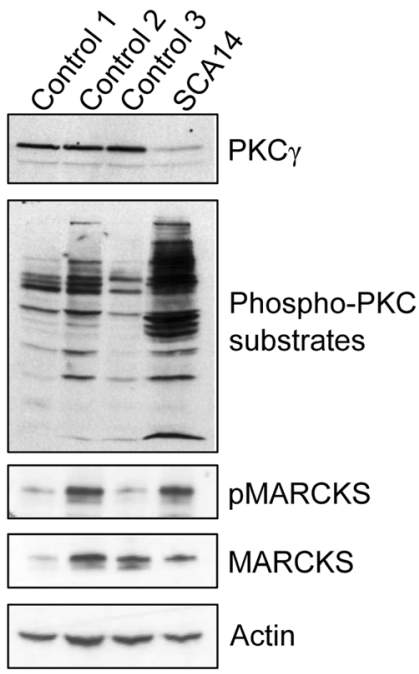

B
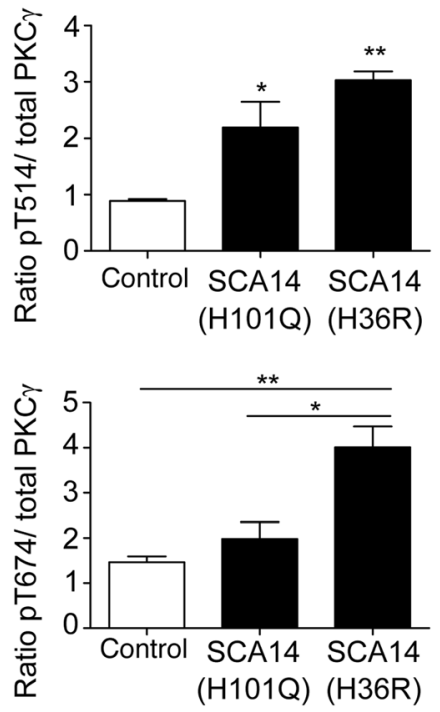

D

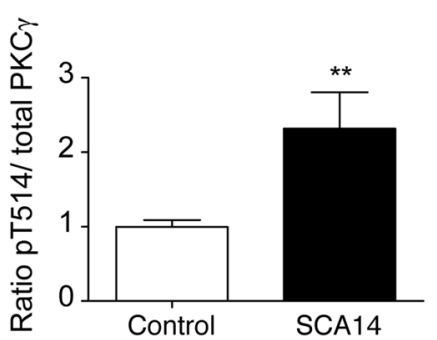


(See figure on previous page.)

Fig. 6 Increased PKC kinase activity in SCA14 patient cells. a Lysates of iPSCs were subjected to immunoblotting for PKCY, T514- and T674phosphorylated PKCY and phospho-PKC substrates. Actin was used as loading control. b Quantification of PKCY phosphorylation at T514 (upper panel) and T674 (lower panel) versus total PKCY. Phosphorylation was significantly increased in SCA14 iPSCs compared to controls $\left(n=3,{ }^{*} p<0.05,{ }^{*} p<0.01\right.$, ANOVA followed by Bonferroni's post-hoc test). $\mathbf{c}$ Lysates from post-mortem cerebellum were subjected to immunoblotting for PKC, , T514-phosphorylated PKC $\gamma$, Calbindin and Actin. $\mathbf{d}$ Quantification of PKC phosphorylation at T514 versus total PKC $\gamma$. T514 phosphorylation was significantly increased in SCA14 (H101Q) cerebellum compared to controls $\left(n=3,{ }^{* *} p<0.01\right.$, unpaired students' t-test). e Cerebellar lysates were subjected to immunoblotting for PKC $\gamma$, phospho-PKC substrates, phosphorylated (p) MARCKS, MARCKS and Actin

been reported previously [11]. Other than the loss of Purkinje cells, little pathology was observed, likely due to the insufficient quality of the post-mortem material. Remaining Purkinje cells were shown to display markedly reduced immunoreactivity for $\mathrm{PKC} \gamma$ without any visible protein aggregation (16). In contrast, our results suggest that aggregation of $\mathrm{PKC} \gamma$ is central to SCA14 pathology. Both mutations investigated in this study cause aggregation of $\mathrm{PKC} \gamma$ in the cytoplasm in both iPSCs and in Purkinje cells of SCA14 (H101Q) cerebellum, but not in other brain regions (which show only minimal $\mathrm{PKC} \gamma$ expression in adult human brain). The aggregation of misfolded proteins is a central feature in many neurodegenerative disorders. Owing to their post-mitotic nature, neurons are particularly vulnerable to misfolded proteins as they cannot dilute toxic substances by division [14]. Moreover, in many neurodegenerative disorders components of the protein degradation machinery are impaired, a phenomenon that is further worsened as neurons age. Interestingly, we found that mutant aggregated $\mathrm{PKC} \gamma$ did not co-localize with ubiquitin. Dephosphorylation of PKC is a prerequisite of the subsequent ubiquitination and degradation via a proteasome pathway [28]. Given the hyper-phosphorylated state of mutant $\mathrm{PKC} \gamma$, our results raise the possibility that mutant $\mathrm{PKC} \gamma$ might be resistant to ubiquitination and subsequent proteasomal or autophagic degradation, which is in contrast to a previous study employing transient overexpression of mutant $\mathrm{PKC} Y$ [46]. This discrepancy might be explained by the massive overexpression of mutant $\mathrm{PKC} \gamma$ that could trigger a cellular response that is different from that under physiological circumstances. We found that there was a significant overlap between PKCY and LC3 in unstimulated SCA14 iPSCs, consistent with the idea that mutant $\mathrm{PKC \gamma}$ is already in an active and aggregated conformation [21, 42]. However, this overlap did not further increase following phorbol ester activation, despite the significant increase in aggregation size. This is consistent with the observation that autophagosomes mostly degrade non-aggregated or small aggregated proteins, but not large inclusions [31]. The enhanced formation of lysosomes in SCA14 iPSCs and cerebellum suggests that mutant $\mathrm{PKC} \gamma$ might enter the lysosomal pathway via alternative routes. Dephosphorylation- and ubiquitination-independent downregulation through lipid raft-mediated endocytic and lysosomal
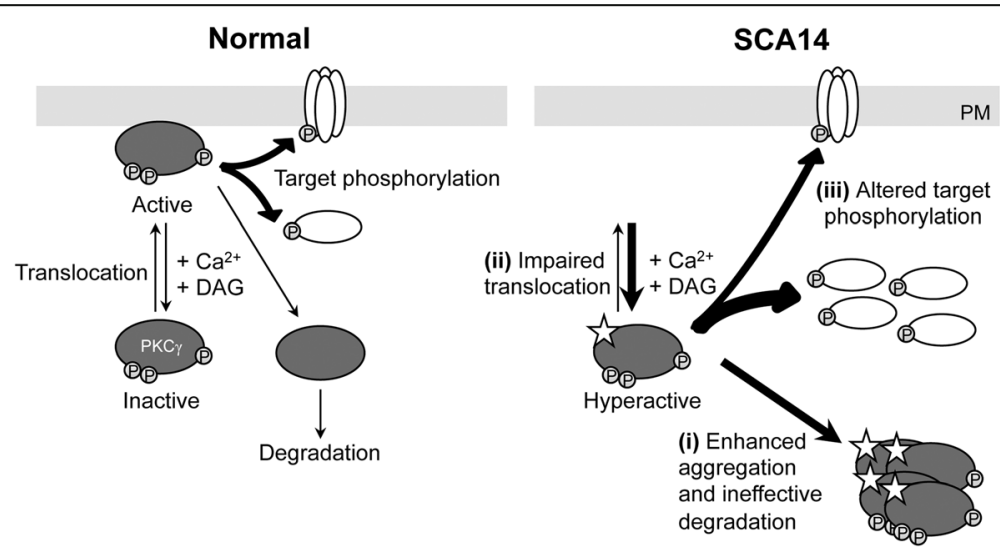

Fig. 7 Model of the functional effect of PKCY mutations. Normally (left panel), mature wildtype PKC resides in the cytosol in an autoinhibited conformation. Binding of diacylglycerol (DAG) and calcium ions $\left(\mathrm{Ca}^{2+}\right)$ activates and promotes the translocation of PKC to the plasma membrane (PM), where active PKCY phosphorylates its membrane substrates. PKC $\gamma$ returns to an autoinhibited conformation (inactive) following the decay of its second messengers. The membrane-bound conformation of PKCY is sensitive to dephosphorylation. Prolonged activation of PKCY leads to its dephosphorylation by phosphatases. The dephosphorylated PKCY can be tagged by ubiquitin and subsequently degraded. In contrast, in SCA14, PKC $y$ with mutated C1 domain adapts an open conformation and is hyper-active in the cytoplasm. (i) Highly phosphorylated mutant PKC $Y$ forms aggregates, which accumulate in the cytoplasm due to inefficient degradation. (ii) Mutant PKCY fails to translocation to the plasma membrane and remains in the cytoplasm. (iii) This might lead to altered phosphorylation of its substrates at the membrane and in the cytoplasm 
pathways has been described for PKC $\alpha$ [27, 29]. Although endosomal sequestration of mutant $\mathrm{PKC} \gamma$ has been observed in vitro [17], this could not be confirmed in our study suggesting the existence of alternative mechanisms such as chaperone-mediated autophagy via LAMP2A [14].

In our study, we found reduced staining of mutant $\mathrm{PKC} \gamma$ at the Purkinje cell membrane in SCA14 cerebellum. This is consistent with previous studies that have suggested altered translocation of mutant $\mathrm{PKC} \gamma$ to the plasma membrane following activation in heterologous cell lines [1, 42] and primary Purkinje cells [39]. PKC has long been implicated in the regulation of neurotransmission and synaptic plasticity by phosphorylating membrane receptors and ion channels [8]. Mutant PKCY might affect membrane excitability in Purkinje cells either indirectly through altering the membrane kinetics of PKC $\alpha$ [39] or directly via phosphorylation of critical receptors. Specific physiological targets of $\mathrm{PKC} \gamma$ remain largely unknown, and their identification might provide important clues about the selective vulnerability of Purkinje cells in SCA14. One possible membrane-associated substrate of $\mathrm{PKC} \gamma$ might be the C3-type transient receptor potential (TRPC3) channel, which is highly expressed in Purkinje cells [19]. In Purkinje cells, TRPC3 is activated downstream of mGluR1 signaling, resulting in calcium influx [19]. Interestingly, $\mathrm{PKC} \gamma$, which is also activated downstream of mGluR1 [23], has been shown to negatively regulate calcium entry via phosphorylation of TRPC3 [40, 41]. Moreover, transiently overexpressed PKCY mutants failed to phosphorylate TRPC3 despite their high catalytic activity [1]. Thus, a failure of mutated PKCY to phosphorylate and inhibit TRPC3 might lead to excessive calcium influx upon TRPC3 activation and might thereby contribute to Purkinje cell dysfunction and cell death. Abnormal TRPC3 signaling is likely to be a common pathological mechanisms in different subtypes of ataxia [4, 30], suggesting a pathological role for $\mathrm{PKC} \gamma$ beyond SCA14. Indeed, Trpc3 and Prkcg were recently identified as hub genes in gene networks misregulated in mouse models of SCA1 [20] and SCA2 [34].

Together, these findings suggest that SCA14 pathogenesis might be partially explained by a loss-of-function of $\mathrm{PKC} Y$ at the cell membrane. However, the absence of SCA14-related phenotypes in Prkcg knockout mice suggests that the disease cannot be fully explained by a reduction of $\mathrm{PKC} \gamma$ function. Interestingly, we found an increase in PKC substrate phosphorylation in SCA14 cerebellum and iPSCs. Increased kinase activity of mutant $\mathrm{PKC} \gamma$ has also been reported in in vitro studies [1, 3, 43], supporting and support the hypothesis that mutations in the $\mathrm{C} 1 \mathrm{do}-$ main facilitate the ligand-induced 'open' and signaling competent conformation of PKC $\gamma$. Recently, increased PKC kinase activity has been shown to be neuroprotective in mouse models of SCA1 and SCA2 [13]. The identities of the phosphorylated PKC targets in these conditions compared to SCA14 remain to be elucidated. Similarly, it will be important to test whether the same aberrantly phosphorylated targets are found in both SCA14 cerebellum and iPSCs. Moreover, it is conceivable that PKC isoforms other than $\mathrm{PKC} \gamma$ contribute to the observed increased PKC substrate phosphorylation. Human stem cells express both canonical and non-canonical PKC isoforms [24] and Purkinje cells have been shown to express $\mathrm{PKC} \alpha, \mathrm{PKC} \gamma, \mathrm{PKC} \delta$ and $\mathrm{PKC} \varepsilon$ [45].

\section{Conclusions}

Our study is the first to describe the functional neuropathology of SCA14 in post-mortem cerebellum as well as in human iPSCs derived from patients with SCA14 mutations. Unexpectedly, $\mathrm{PKC} \gamma$ aggregation, mislocalization and increased kinase activity that we observed in SCA14 cerebellum were reproduced in SCA14 iPSCs. Purkinje cells are particularly vulnerable in SCA14, likely due to their high expression of $\mathrm{PKC} \gamma$ and its specific targets that regulate the calcium homeostasis and the unique physiological properties of these neurons. While the latter cannot be modelled in undifferentiated stem cells, the fact that patient iPSCs express $\mathrm{PKC} \gamma$ and recapitulate key pathological findings observed in SCA14 cerebellum underscores their potential as relevant tools for disease modeling and drug discovery, in addition to future studies in which SCA14 iPSCs will be differentiated to Purkinje cells.

\section{Additional file}

Additional file 1: Supplementary Material. (PDF $3471 \mathrm{~kb}$ )

\section{Abbreviations}

DAG: Diacylglycerol; iPSCs: Induced pluripotent stem cells; LAMP2: Lysosomeassociated membrane protein 2; LC3: Microtubule-associated protein 1 light chain 3; MARCKS: Myristoylated alanine-rich C-kinase substrate; PDBu: Phorbol 12, 13-dibutyrate; PKCY: Protein kinase C gamma; PMA: Phorbol 12-myristate 13-acetate; SCA14: Spinocerebellar ataxia type 14; UPS: Ubiquitin proteasome system

\section{Acknowledgments}

We are immensely grateful to all patients for their participation. We acknowledge the Oxford Parkinson's Disease Center (OPDC) study for the original generation of iPSC lines from control donors, funded by the Monument Trust Discovery Award from Parkinson's UK, a charity registered in England and Wales (2581970) and in Scotland (SC037554), with the support of the National Institute for Health Research (NIHR) Oxford Biomedical Research Center based at Oxford University Hospitals NHS Trust and University of Oxford, and the NIHR Comprehensive Local Research Network. Human peripheral blood mononuclear cells (PBMCs) were a generous gift from Professor Quentin Sattentau, University of Oxford. We thank the High-Throughput Genomics Group at the Wellcome Trust Centre for Human Genetics, Oxford (Funded by Wellcome Trust grant reference 090532/Z/09/Z and MRC Hub grant G0900747 91070) for the generation of Illumina genotyping and transcriptome data for characterization of iPSC lines. We also acknowledge the Oxford Brain Bank, supported by the UK Medical Research Council and Alzheimer's Brain Bank UK. 


\section{Funding}

Supported by the Royal Society (E.B.E.B.), Ataxia UK (E.B.E.B.), the European Union's Horizon 2020 research and innovation program (under the Marie Skłodowska-Curie grant agreement no. 699978) (L.M.W.), the John Fell OUP Fund (E.B.E.B., L.M.W.) and the Monument Trust Discovery Award from Parkinson's UK (J.V.) and the Oxford NIHR Biomedical Research Centre (O.A.) This publication reflects the views only of the authors, and the European Commission cannot be held responsible for any use, which may be made of the information contained therein. The Wellcome Trust (WTISSF121302) and The Oxford Martin School (LC0910-004) provide financial support to the James Martin Stem Cell Facility (S.A.C.). The funding bodies had no role in the design of the study and collection, analysis, and interpretation of data and in writing the manuscript.

\section{Availability of data and materials}

All data generated and analyzed during this study are included in the published article [and its supplementary information files].

\section{Authors' contributions}

MMKW carried out IPSC reprogramming and all functional experiments in iPSCs, designed and interpreted experiments, and was a major contributor in writing the manuscript. SDH, JV, LMW and SAC were involved in IPSC reprogramming. GF and AHN identified and diagnosed SCA14(H36R) patients. OA carried out neuropathology experiments and interpreted the patient histopathology. KT identified and diagnosed SCA14(H101Q) patients, interpreted findings and helped writing the manuscript. EBEB performed biochemical experiments on SCA14 cerebellum, designed and interpreted experiments and wrote the manuscript. All authors read and approved the final manuscript.

\section{Ethics approval and consent to participate}

The human iPSC lines used for this study were derived from human skin biopsy fibroblasts, following signed informed consent, with approval from the National Research Ethics Service Committee, South Central - Southampton B (REC reference number 12/SC/0106). Human tissues were collected and studied under REC reference 15/SC/0639 of the Oxford Brain Bank, an HTA-licensed research tissue bank of the University of Oxford. The human PBMCs used for this study were obtained following signed informed consent, with approval from the West Midlands - Coventry and Warwickshire Research Ethics Committee (REC reference number 17/WM/0333).

\section{Consent for publication}

Consent for publication has been obtained.

\section{Competing interests}

The authors declare that they have no competing interests.

\section{Publisher's Note}

Springer Nature remains neutral with regard to jurisdictional claims in published maps and institutional affiliations.

\section{Author details}

'Department of Physiology, Anatomy and Genetics, University of Oxford, Sherrington Road, Oxford OX1 3PT, UK. ${ }^{2}$ Sir William Dunn School of Pathology, University of Oxford, South Parks Road, Oxford OX1 3RE, UK. ${ }^{3}$ Gloucestershire Hospitals, NHS Foundation Trust, Cheltenham General Hospital, Sandford Road, Cheltenham GL53 7AN, UK. ${ }^{4}$ Nuffield Department of Clinical Neurosciences, University of Oxford, Level 6, West Wing, John Radcliffe Hospital, Oxford OX3 9DU, UK. ${ }^{5}$ Oxford Centre for Genomic Medicine, ACE Building, Oxford University Hospitals NHS Trust, Nuffield Orthopaedic Centre, Windmill Road, Oxford OX3 7HE, UK.

Received: 13 September 2018 Accepted: 14 September 2018 Published online: 24 September 2018

\section{References}

1. Adachi N, Kobayashi T, Takahashi H, Kawasaki T, Shirai Y, Ueyama T, Matsuda T, Seki T, Sakai N, Saito N (2008) Enzymological analysis of mutant protein kinase $C_{Y}$ causing spinocerebellar ataxia type 14 and dysfunction in $\mathrm{Ca} 2+$ homeostasis. J Biol Chem 283:19854-19863. https://doi.org/10.1074/jbc. M801492200
2. Antal CE, Newton AC (2014) Tuning the signalling output of protein kinase C. Biochem Soc Trans 42:1477-1483. https://doi.org/10.1042/BST20140172

3. Asai H, Hirano M, Shimada K, Kiriyama T, Furiya Y, Ikeda M, Iwamoto T, Mori T, Nishinaka K, Konishi N, Udaka F, Ueno S (2009) Protein kinase C gamma, a protein causative for dominant ataxia, negatively regulates nuclear import of recessive-ataxia-related aprataxin. Hum Mol Genet 18:3533-3543. https:// doi.org/10.1093/hmg/ddp298

4. Becker EBE (2017) From mice to men: TRPC3 in cerebellar Ataxia. Cerebellum 16:877-879. https://doi.org/10.1007/s12311-015-0663-y

5. Beers J, Gulbranson DR, George N, Siniscalchi LI, Jones J, Thomson JA, Chen $\mathrm{G}$ (2012) Passaging and colony expansion of human pluripotent stem cells by enzyme-free dissociation in chemically defined culture conditions. Nat Protoc 7:2029-2040. https://doi.org/10.1038/nprot.2012.130

6. Bolte S, Cordelières FP (2006) A guided tour into subcellular colocalization analysis in light microscopy. J Microsc 224:213-232. https://doi.org/10.1111/ j.1365-2818.2006.01706.x

7. Brkanac Z, Bylenok L, Fernandez M, Matsushita M, Lipe H, Wolff J, Nochlin D, Raskind WH, Bird TD (2002) A new dominant spinocerebellar ataxia linked to chromosome 19q13.4-qter. Arch Neurol 59:1291-1295

8. Callender JA, Newton AC (2017) Conventional protein kinase C in the brain: 40 years later. Neuronal Signal 1:NS20160005-NS20160010. https://doi.org/ 10.1042/NS20160005

9. Chelban V, Wiethoff S, Fabian-Jessing BK, Haridy NA, Khan A, Efthymiou S, Becker EBE, O'Connor E, Hersheson J, Newland K, Hojland AT, Gregersen PA, Lindquist SG, Petersen MB, Nielsen JE, Nielsen M, Wood NW, Giunti P, Houlden H (2018) Genotype-phenotype correlations, dystonia and disease progression in spinocerebellar ataxia type 14. Mov Disord. https://doi.org/10. 1002/mds.27334

10. Chen C, Kano M, Abeliovich A, Chen L, Bao S, Kim JJ, Hashimoto K, Thompson RF, Tonegawa S (1995) Impaired motor coordination correlates with persistent multiple climbing fiber innervation in PKC gamma mutant mice. Cell 83:1233-1242

11. Chen D-H, Brkanac Z, Verlinde CLMJ, Tan X-J, Bylenok L, Nochlin D, Matsushita M, Lipe H, Wolff J, Fernandez M, Cimino PJ, Bird TD, Raskind WH (2003) Missense mutations in the regulatory domain of PKC gamma: a new mechanism for dominant nonepisodic cerebellar ataxia. Am J Hum Genet 72:839-849

12. Chen D-H, Raskind WH, Bird TD (2012) Spinocerebellar ataxia type 14 Handb Clin Neurol 103:555-559. https://doi.org/10.1016/B978-0-444-51892-7. 00036-X

13. Chopra R, Wasserman AH, Pulst SM, De Zeeuw Cl, Shakkottai VG (2018) Protein kinase $\mathrm{C}$ activity is a protective modifier of Purkinje neuron degeneration in cerebellar ataxia. Hum Mol Genet 27:1396-1410. https://doi. org/10.1093/hmg/ddy050

14. Ciechanover A, Kwon YT (2017) Protein quality control by molecular chaperones in Neurodegeneration. Front Neurosci 11:185. https://doi.org/10. 3389/fnins.2017.00185

15. Curnutte JT, Erickson RW, Ding J, Badwey JA (1994) Reciprocal interactions between protein kinase $\mathrm{C}$ and components of the NADPH oxidase complex may regulate superoxide production by neutrophils stimulated with a phorbol ester. J Biol Chem 269:10813-10819

16. Dafinca R, Scaber J, Ababneh N, Lalic T, Weir G, Christian H, Vowles J, Douglas AGL, Fletcher-Jones A, Browne C, Nakanishi M, Turner MR, WadeMartins R, Cowley SA, Talbot K (2016) C9orf72 Hexanucleotide expansions are associated with altered endoplasmic reticulum calcium homeostasis and stress granule formation in induced pluripotent stem cell-derived neurons from patients with amyotrophic lateral sclerosis and Frontotemporal dementia. Stem Cells 34:2063-2078. https://doi.org/10.1002/stem.2388

17. Doran G, Davies KE, Talbot K (2008) Activation of mutant protein kinase Cgamma leads to aberrant sequestration and impairment of its cellular function. Biochem Biophys Res Commun 372:447-453. https://doi.org/10. 1016/j.bbrc.2008.05.072

18. Handel AE, Chintawar S, Lalic T, Whiteley E, Vowles J, Giustacchini A, Argoud K, Sopp P, Nakanishi M, Bowden R, Cowley S, Newey S, Akerman C, Ponting CP, Cader MZ (2016) Assessing similarity to primary tissue and cortical layer identity in induced pluripotent stem cell-derived cortical neurons through single-cell transcriptomics. Hum Mol Genet 25:989-1000. https://doi.org/10. 1093/hmg/ddv637

19. Hartmann J, Dragicevic E, Adelsberger H, Henning HA, Sumser M, Abramowitz J, Blum R, Dietrich A, Freichel M, Flockerzi V, Birnbaumer L, Konnerth A (2008) TRPC3 channels are required for synaptic transmission 
and motor coordination. Neuron 59:392-398. https://doi.org/10.1016/j. neuron.2008.06.009

20. Ingram M, Wozniak EAL, Duvick L, Yang R, Bergmann P, Carson R, O'Callaghan B, Zoghbi HY, Henzler C, Orr HT (2016) Cerebellar Transcriptome profiles of ATXN1 transgenic mice reveal SCA1 disease progression and protection pathways. Neuron 89:1194-1207. https://doi. org/10.1016/j.neuron.2016.02.011

21. Jezierska J, Goedhart J, Kampinga HH, Reits EA, Verbeek DS (2013) SCA14 mutation V138E leads to partly unfolded PKC $\gamma$ associated with an exposed C-terminus, altered kinetics, phosphorylation and enhanced insolubilization. J Neurochem 128:741-751. https://doi.org/10.1111/jnc.12491

22. Kano M, Hashimoto $\mathrm{K}$, Chen $\mathrm{C}$, Abeliovich A, Aiba A, Kurihara H, Watanabe M, Inoue Y, Tonegawa S (1995) Impaired synapse elimination during cerebellar development in PKCY mutant mice. Cell 83:1223-1231. https:// doi.org/10.1016/0092-8674(95)90147-7

23. Kano M, Watanabe T (2017) Type-1 metabotropic glutamate receptor signaling in cerebellar Purkinje cells in health and disease. F1000Res 6:416. https://doi.org/10.12688/f1000research.10485.1

24. Kinehara M, Kawamura S, Tateyama D, Suga M, Matsumura H, Mimura S, Hirayama N, Hirata M, Uchio-Yamada K, Kohara A, Yanagihara K, Furue MK (2013) Protein kinase $C$ regulates human pluripotent stem cell self-renewal. PLoS One 8:e54122-e54113. https://doi.org/10.1371/journal.pone.0054122

25. Koeppen AH (2005) The pathogenesis of spinocerebellar ataxia. Cerebellum 4:62-73. https://doi.org/10.1080/14734220510007950

26. Kose A, Saito N, Ito H, Kikkawa U, Nishizuka Y, Tanaka C (1988) Electron microscopic localization of type I protein kinase $\mathrm{C}$ in rat Purkinje cells. J Neurosci 8:4262-4268

27. Leontieva OV, Black JD (2004) Identification of two distinct pathways of protein kinase Calpha down-regulation in intestinal epithelial cells. J Biol Chem 279:5788-5801. https://doi.org/10.1074/jbc.M308375200

28. Lu Z, Liu D, Hornia A, Devonish W, Pagano M, Foster DA (1998) Activation of protein kinase $C$ triggers its ubiquitination and degradation. Mol Cell Biol 18:839-845

29. Lum MA, Pundt KE, Paluch BE, Black AR, Black JD (2013) Agonist-induced down-regulation of endogenous protein kinase $\mathrm{c}$ a through an endolysosomal mechanism. J Biol Chem 288:13093-13109. https://doi.org/ 10.1074/jbc.M112.437061

30. Meera P, Pulst SM, Otis TS (2016) Cellular and circuit mechanisms underlying spinocerebellar ataxias. J Physiol 594:4653-4660. https://doi.org/ 10.1113/JP271897

31. Menzies FM, Fleming A, Caricasole A, Bento CF, Andrews SP, Ashkenazi A, Füllgrabe J, Jackson A, Jimenez Sanchez M, Karabiyik C, Licitra F, Lopez Ramirez A, Pavel M, Puri C, Renna M, Ricketts T, Schlotawa L, Vicinanza M, Won H, Zhu Y, Skidmore J, Rubinsztein DC (2017) Autophagy and Neurodegeneration: pathogenic mechanisms and therapeutic opportunities. Neuron 93:1015-1034. https://doi.org/10.1016/j.neuron.2017.01.022

32. Németh AH, Kwasniewska AC, Lise S, Parolin Schnekenberg R, EBE B, Bera KD, Shanks ME, Gregory L, Buck D, Zameel Cader M, Talbot K, de Silva R, Fletcher N, Hastings R, Jayawant S, Morrison PJ, Worth P, Taylor M, Tolmie J, O'Regan M, Ataxia Consortium UK, Valentine R, Packham E, Evans J, Seller A, Ragoussis J (2013) Next generation sequencing for molecular diagnosis of neurological disorders using ataxias as a model. Brain 136:3106-3118. https://doi.org/10.1093/brain/awt236

33. Nixon JB, McPhail LC (1999) Protein kinase C (PKC) isoforms translocate to tritoninsoluble fractions in stimulated human neutrophils: correlation of conventional PKC with activation of NADPH oxidase. J Immunol 163:4574-4582

34. Pflieger LT, Dansithong W, Paul S, Scoles DR, Figueroa KP, Meera P, Otis TS, Facelli JC, Pulst SM (2017) Gene co-expression network analysis for identifying modules and functionally enriched pathways in SCA2. Hum Mol Genet 14:269-212. https://doi.org/10.1093/hmg/ddx191

35. Ross CA, Poirier MA (2005) Opinion: what is the role of protein aggregation in neurodegeneration? Nat Rev MolCell Bio 6:891-898. https://doi.org/10. 1038/nrm1742

36. Seki T, Adachi N, Ono Y, Mochizuki H, Hiramoto K, Amano T, Matsubayashi H, Matsumoto M, Kawakami H, Saito N, Sakai N (2005) Mutant protein kinase Cgamma found in spinocerebellar ataxia type 14 is susceptible to aggregation and causes cell death. J Biol Chem 280:29096-29106. https:// doi.org/10.1074/jbc.M501716200

37. Seki T, Shimahara T, Yamamoto K, Abe N, Amano T, Adachi N, Takahashi H, Kashiwagi K, Saito N, Sakai N (2009) Mutant gammaPKC found in spinocerebellar ataxia type 14 induces aggregate-independent maldevelopment of dendrites in primary cultured Purkinje cells. Neurobiol Dis 33:260-273. https://doi.org/10.1016/j.nbd.2008.10.013

38. Seki T, Takahashi H, Adachi N, Abe N, Shimahara T, Saito N, Sakai N (2007) Aggregate formation of mutant protein kinase $\mathrm{C}$ gamma found in spinocerebellar ataxia type 14 impairs ubiquitin-proteasome system and induces endoplasmic reticulum stress. Eur J Neurosci 26:3126-3140. https:// doi.org/10.1111/j.1460-9568.2007.05933.x

39. Shuvaev AN, Horiuchi H, Seki T, Goenawan H, Irie T, lizuka A, Sakai N, Hirai H (2011) Mutant PKCY in spinocerebellar ataxia type 14 disrupts synapse elimination and long-term depression in Purkinje cells in vivo. J Neurosci 31 14324-14334. https://doi.org/10.1523/JNEUROSCI.5530-10.2011

40. Trebak M, Hempel N, Wedel BJ, Smyth JT, Bird GSJ, Putney JW (2005) Negative regulation of TRPC3 channels by protein kinase C-mediated phosphorylation of serine 712. Mol Pharmacol 67:558-563. https://doi.org/ 10.1124/mol.104.007252

41. Venkatachalam K (2003) Regulation of canonical transient receptor potential (TRPC) channel function by diacylglycerol and protein kinase C. J Biol Chem 278:29031-29040. https://doi.org/10.1074/jbc.M302751200

42. Verbeek DS, Goedhart J, Bruinsma L, Sinke RJ, Reits EA (2008) PKC gamma mutations in spinocerebellar ataxia type 14 affect C1 domain accessibility and kinase activity leading to aberrant MAPK signaling. I Cell Sci 121:23392349. https://doi.org/10.1242/jcs.027698

43. Verbeek DS, Knight MA, Harmison GG, Fischbeck KH, Howell BW (2005) Protein kinase C gamma mutations in spinocerebellar ataxia 14 increase kinase activity and alter membrane targeting. Brain 128:436-442. https://doi. org/10.1093/brain/awh378

44. Watson LM, Wong MMK, Vowles J, Cowley SA, Becker EBE (2018) A simplified method for generating Purkinje cells from human-induced pluripotent stem cells. Cerebellum 17:419-427. https://doi.org/10.1007/ s12311-017-0913-2

45. Wetsel WC, Khan WA, Merchenthaler I, Rivera H, Halpern AE, Phung HM, Negro-Vilar A, Hannun YA (1992) Tissue and cellular distribution of the extended family of protein kinase C isoenzymes. J Cell Biol 117:121-133. https://doi.org/10.1083/jcb.117.1.121

46. Yamamoto K, Seki T, Adachi N, Takahashi T, Tanaka S, Hide I, Saito N, Sakai N (2010) Mutant protein kinase C gamma that causes spinocerebellar ataxia type 14 (SCA14) is selectively degraded by autophagy. Genes Cells 15:425438. https://doi.org/10.1111/j.1365-2443.2010.01395.x

Ready to submit your research? Choose BMC and benefit from:

- fast, convenient online submission

- thorough peer review by experienced researchers in your field

- rapid publication on acceptance

- support for research data, including large and complex data types

- gold Open Access which fosters wider collaboration and increased citations

- maximum visibility for your research: over $100 \mathrm{M}$ website views per year

At BMC, research is always in progress.

Learn more biomedcentral.com/submissions 